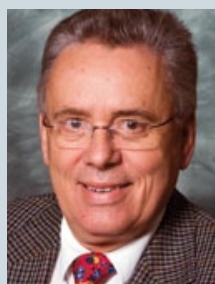

Dr. med.

Hartmut Koch,

Vechta

\section{Honig als Hustenmittel evidenzbasiert}

Honig ist ein altes Hausmittel gegen Husten. Obwohl es in diesem Zusammenhang sogar von der Weltgesundheitsorganisation empfohlen wird, fehlte bislang eine Evidenz für die Wirkung fast völlig. Eine Arbeitsgruppe aus Israel legt jetzt eine doppelblinde, randomisierte, placebokontrollierte Studie zur Wirkung von Honig als Hustenmittel bei Kindern im Alter zwischen ein und fünf Jahren vor.

W issenschaftler nahmen 300 Kinder mit oberen Atemwegsinfektionen von weniger als sieben Tagen Dauer in die Studie auf. Patienten mit Asthma, Pneumonie, Kruppsyndrom, Sinusitis und allergischer Rhinitis wurden ausgeschlossen. Für die Bestimmung des Schweregrades des Hustens wurde den Eltern ein validierter Fragebogen aus fünf Fragen vorgelegt. Es wurden drei verschiedene Sorten Honig verwendet: Eukalyptushonig, Honig aus Lippenblütengewächsen (z.B. Lavendel) und Zitrushonig. Als Placebo wurde ein Sirup aus Dattelextrakt eingesetzt.

Die Probanden erhielten vor dem Schlafengehen $10 \mathrm{~g}$ der jeweiligen Honigsorte oder Placebo. Am folgenden Tag wurden die Eltern von einem geblindeten Interviewer befragt unter Verwendung derselben Fragen, die zuvor schon einmal schriftlich gestellt worden waren. Die Kinder waren zum Zeitpunkt der Studie im Durchschnitt drei Tage krank.

Es zeigte sich, dass bei jeder Honigsorte das Ansprechen verglichen mit dem Dattelsirup deutlich besser war. Zwischen den drei Honigsorten gab es keine Unterschiede. Der Husten sei weniger quälend gewesen. Die Kinder haben auch besser geschlafen. Auch unter Anwendung des Dattelsirups kam es zu einer Besserung.
Eine ähnliche Studie von Paul et al. [Arch Pediatr Adoles Med 2007; 161: 1140-6], bei der Honig mit Dextromethorphan und keiner Behandlung verglichen wurde, hatte ebenfalls den Nutzen von Honig gezeigt. Über den Wirkungsmechanismus kann nur spekuliert werden: Eventuell liegt es an den enthaltenen Antioxidanzien.

Sicher feststellen lässt sich, dass Honig wesentlich ungefährlicher ist als andere, in vielen Ländern nicht verschreibungspflichtige Inhaltsstoffe von Hustenmitteln für Kleinkinder wie Pseudoephedrin, Dextromethorphan und Diphenhydramin. Die Autoren weisen ausdrücklich darauf hin, dass Honig Kindern unter einem Jahr wegen des Risikos des Säuglingsbotulismus nicht verabfolgt werden soll. Wegen der Kariesgefahr warnen die Autoren vor jeder länger dauernden Anwendung.

Cohen HA et al. Effect of honey on nocturnal cough and sleep quality: A double-blind, randomized, placebo-controlled study. Pediatrics 2012; 130: 465-71

Kommentar: Es ist hocherfreulich, dass mit dieser Studie ein altes, billiges, praktisch überall zur Verfügung stehendes Hausmittel zur symptomatischen Behandlung oberer Atemwegsinfektionen bei Kleinkindern eine Evidenzbasierung erfährt. Es darf nicht

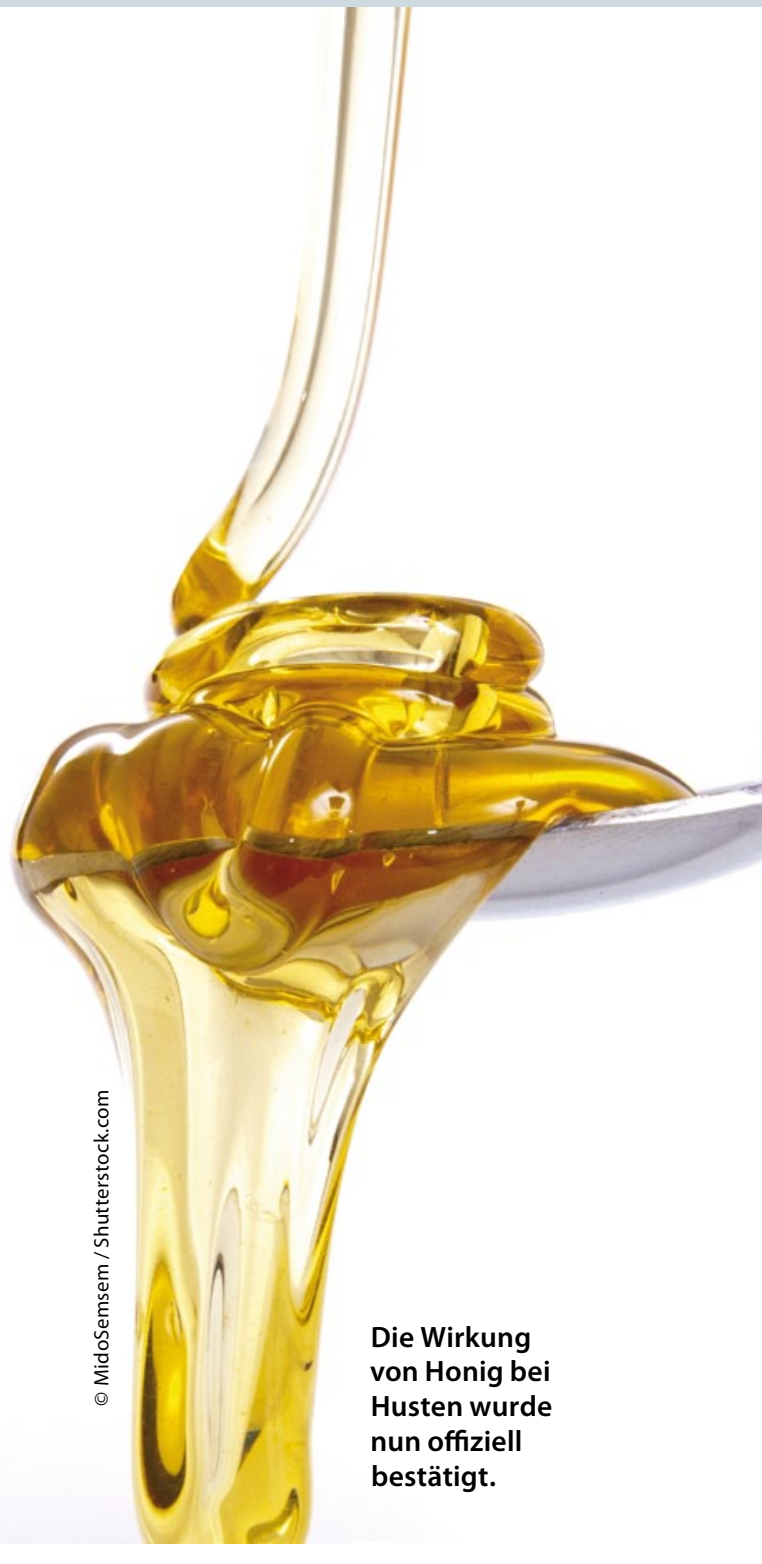

vergessen werden, dass dieses Mittel wesentlich billiger ist als jedes andere in der Apotheke für diese Beschwerden erhältliche. Möglicherweise lassen allerdings Einfachheit der Anwendung und der geringe Preis viele Eltern an der Wirkung zweifeln.

Dr. Hartmut Koch 